\title{
Twitter, \#primaveravalenciana y generación de noticias
}

\author{
Jaime ALBERO-GABRIEL* \\ jaime.albero@gmail.com
}

(Abstracts y palabras clave al final del artículo)

Enviado: 20 de abril de 2013

Evaluado: 10 de junio de 2013

Aceptado: 14 de julio de 2013

\section{INTRODUCCIÓN}

En la presente investigación se pretende contribuir a comprender la profunda interrelación que existe en la generación y modificación de contenidos de la Agenda Setting, y de la Opinión Pública en general, gracias a la aparición de redes de microblogging con carácter social como es el caso de Twitter. Los objetos de estudio son de difícil definición ya que, en el primer caso, las investigaciones sobre Opinión Pública no han llegado a delimitar definitivamente el concepto (o bien lo han tratado como un resultado de interrelaciones sujetas a infinidad de condicionantes), mientras que en el segundo, la relativamente reciente aparición de las plataformas de microblogging no ha generado todavía una suficiente cantidad de estudios científicos y académicos sobre su capacidad, usos y efectos en nuevas formas de comunicación en la sociedad.

Estos condicionantes hacen que el investigador se plantee objetivos modestos en su trabajo: el análisis de la penetración del uso de Twitter entre los profesionales de la generación de noticias, el uso que de las redes de microblogging hacen los entes tradicionalmente emisores de contenidos noticiables, la respuesta por parte de los receptores que estas emisiones en Twitter generan y los efectos que pueden llegar a tener, así como una previsión de las tendencias de futuro (si las hubiera) en el uso de dicha red por parte de los actores del proceso comunicativo.

Una de las dificultades añadidas al planteamiento de esta investigación es la escasez de publicaciones académicas referidas a este tipo de redes y sus efectos en la sociedad y en procesos sociales como la formación de la Opinión Pública y el establecimiento de la Agenda Setting. La posible influencia de Twitter es difícilmente mensurable, dado que apenas se ha sistematizado la forma de medición cuantitativa de las publicaciones efectuadas en dicha red, ni se ha entrado a la ingente

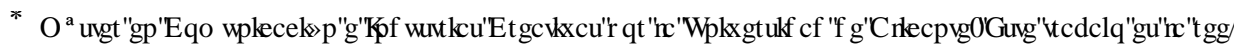

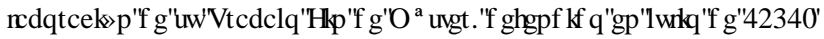


tarea de realizar un análisis de discurso de dichas publicaciones. De la misma manera, no se conoce el grado de influencia que puede llegar a tener en los medios de comunicación, y los efectos (tanto positivos como negativos) que desde la perspectiva de calidad comunicativa puede acarrear en el día a día de las redacciones de los diarios de prensa regional o de cercanía.

En la presente investigación se pretende constatar o refutar si ha existido una influencia manifiesta sobre los diarios regionales de la provincia de Valencia en la cobertura de los incidentes acaecidos en la capital entre el 15 de febrero de 2012 y el 1 de marzo de 2012 a raíz de las protestas estudiantiles que se han popularizado como \#primaveravalenciana en la red Twitter.

Trataremos de demostrar, mediante triangulación metodológica, que dicha influencia es mensurable y cualitativamente significativa, mediante la medición de términos relacionados con dicha red de microblogging en los artículos (noticias, artículos de opinión y cartas al director) publicados por los diarios de mayor tirada de la provincia de Valencia. En este caso Levante-EMV y Las Provincias.

La influencia de los medios en los públicos a los que se dirige se ha visto matizada por la aparición de nuevos espacios comunicativos colaborativos y multidireccionales, en los que el tradicional consumidor de contenidos informativos generados en los medios de producción política, social y cultural se ha convertido a su vez en prosumidor de información.

Consumers are a phenomenon of the Industrial Age. As society moves toward the Post-Industrial Age, so will the number of pure consumers decline. They will be replaced by "prosumers", people who produce many of their own goods and services (Kotler, 1986, p. 510-513).

En virtud de estos nuevos aspectos, las relaciones entre medios y públicos se han transformado y la influencia resulta ser de doble sentido, influyendo el supuesto influenciado al influenciador, y corroborando que "el poder de los medios puede variar con el tiempo" (McQuail, 2000, p. 501).

Las recientes movilizaciones en el mundo árabe han despertado el interés por este proceso. Pero existe una dificultad intrínseca al medio, que es su reciente aparición. Por una parte, los medios de comunicación atribuyen una gran influencia a las redes sociales en el devenir de las movilizaciones. Por otro lado, la comunidad científica está intentando acotar la influencia real de las TIC en estos movimientos. Para algunos la acción online fue meramente un complemento de la acción offline. No fue una herramienta de empoderamiento de colectivos que tradicionalmente no tenían poder (Aouragh and Alexander, 2011; Morozov, 2010). Pero a pesar de sus limitaciones, las TIC, y especialmente Internet, hacen que las acciones offline y online se refuercen unas a otras (Carty, 2010; Earl et al., 2010; Fisher and Boekkooi, 2010). Además, en el caso de la Primavera Árabe hay que matizar la influencia de las TIC dada la baja penetración de internet y de los dispositivos móviles entre la población para acotar mejor el análisis.

Este cambio de rol del usuario de los medios de comunicación hace también cambiar el rol de los periodistas (Fumero-Reverón, 2011). Se abre así un campo lleno de posibilidades para los profesionales del periodismo, que pueden apoyarse en más fuentes de información que antes (Cohen, Hamilton y Turner, 2011). 


\section{OPINIÓN PÚBLICA}

El concepto de Opinión Pública es difícil de definir y de delimitar dada su alta complejidad operativa. Para Rubio Ferreres "la opinión pública es un fenómeno comunicativo y psicosocial que depende del contexto histórico y sociocultural" (2009: 2). Dicho contexto, en una sociedad marcada por una comunicación unidireccional, era claramente enfocado por los emisores y mediadores de los mensajes. Las emisiones eran después recogidas por institutos de medición de la Opinión Pública que, a través de encuestas, establecían la pregnancia y pervivencia de los mensajes lanzados a la población. De esta manera, en 1973, Pierre Bourdieu afirma que "las problemáticas que se le imponen a este tipo de organismos están profundamente ligadas a la coyuntura y dominadas por un tipo determinado de demanda social" (2000, p. 220).

Mediante una campaña de visibilización, moldeado ideológico y jerarquización de las noticias, los medios de comunicación establecen los temas acerca de los que discutirán las personas en un momento determinado. "La prensa no tiene mucho éxito en decir a la gente qué tiene que pensar pero sí lo tiene en decir a sus lectores sobre qué tienen que pensar" (Cohen, 1963, p. 13).

Las opiniones se forman tras un proceso en el que participan gran diversidad de factores. Las creencias y los valores políticos de los individuos, sus ideas acerca de la sociedad y del sistema político, su nivel de información acerca de las normas, reglas, instituciones y estructuras de autoridad, son elementos importantes en la formación de sus opiniones acerca de las cuestiones políticas. Además de las formulaciones de Foote y Hart y de Deutsch (entendiéndola como proceso unidireccional y desde las elites), otra concepción en la formación de la Opinión Pública es aquella que entiende que funciona de "abajo hacia arriba", según la cual, esta sería un "... rebullir del cuerpo social que sale hacia lo alto..." (Sartori, 1992, p. 153). En este caso, sería la ciudadanía la que proporciona un tema de interés a la agenda de los medios de comunicación. La ciudadanía plantea, discute y propone puntos de vista, elige uno y lo hace visible ante el público general, mandando a sus representantes que cumplan las decisiones obtenidas.

En esta última teoría, Sartori introduce el feedback, completando las teorías anteriores. Señala a los líderes de opinión como actores cruciales en la intermediación entre diversos niveles de opinión. Esta mediación, mediante las nuevas formas de comunicación, se convierte en transversal en todo el proceso de formación de la Opinión Pública. Pero no fue Sartori el primero en darse cuenta de la importancia de los líderes de opinión en el proceso de decisión política.

Los líderes de opinión juegan un rol especial en la red de relaciones personales... las ideas a menudo fluyen desde la radio y la prensa hacia los líderes de opinión y desde ellos hacia los sectores de la población menos activos [...] Las influencia persona-a-persona alcanza a aquellos quienes son más susceptibles al cambio, y sirve como un puente sobre el cual los medios formales de comunicación extienden su influencia (Lazarsfeld, Berelson y Gaudet, 1944, pp. 151-152).

Hay que tener en cuenta también en este punto el papel de los nuevos medios de comunicación, sus rutinas y las audiencias a las que se dirigen. 
Los nuevos medios de comunicación determinan una audiencia segmentada y diferenciada que, aunque masiva en cuanto a su número, ya no es de masas en cuanto a la simultaneidad y uniformidad del mensaje que recibe. Los nuevos medios de comunicación ya no son medios de comunicación de masas en el sentido tradicional de envío de un número limitado de mensajes a una audiencia de masas homogénea. Debido a la multiplicidad de mensajes y fuentes, la propia audiencia se ha vuelto más selectiva. La audiencia seleccionada tiende a elegir sus mensajes, por lo que profundiza su segmentación y mejora la relación individual entre emisor y receptor (Sabbah, p. 219, apud Castells, 2000, p. 412).

Esta segmentación que se anunciaba en el ya lejano año 2000 se ha visto aumentada exponencialmente mediante la aparición de las redes sociales y de microblogging. Complementando el concepto de prosumidor de Toffler, Castells (2009, p. 88) acuña el término "autocomunicación de masas", basado en la viralidad de los contenidos que se difunden mediante internet y en la capacidad de segmentación, tanto de los emisores de los mensajes como de los receptores de los mismos.

\section{AGENDA SETTING}

Nos referimos a la Agenda Setting cuando inferimos una fuerte correlación entre el énfasis que los medios de comunicación ponen en determinados contenidos informativos y la importancia que desde la audiencia se atribuye a esos contenidos. Por su parte, la teoría del Framing o Análisis de Marcos, supone que se puede presentar información compleja de tal manera que el usuario, previamente condicionado por un ambiente personal, social e informativo determinado, saque unas conclusiones comprensibles mediante la utilización de constructos mentales predefinidos. De esta manera:

To frame is to select some aspects of a perceived reality and make them more salient in a communicating text, in such a way as to promote a particular problem definition, causal interpretation, moral evaluation, and/or treatment recommendation for the item described (Entman, 1993, p. 52).

En estudios posteriores, se concibe la teoría del Framing como una extensión de la teoría de la Agenda Setting. Se utiliza el término second-level agenda-setting, que describiría el impacto en la interpretación de las historias noticiables por parte de las audiencias de los medios (McCombs, Shaw y Weaver, 1997).

Desde la década de los 80 se tiende a comprender los efectos de los medios desde el constructivismo social. Tales efectos estarían matizados por la relación entre el emisor y el receptor, lo que refuerza la Teoría del Framing (McQuail, 1994). Desde esta perspectiva, los medios establecen las marcas diferenciales que los lectores utilizan para interpretar la realidad. Paralelamente, existen estructuras y esquemas previos que ayudan al receptor a procesar la información (Tuchman, 1978).

Para esta perspectiva teórica, los individuos buscan alternativas de información, porque se asume que la información dada por los medios de comunicación de masas es incompleta o parcial. En un proceso posterior de reflexión y comparación de informaciones con otros interlocutores de sus ámbitos cercanos, el usuario de los 
medios de comunicación matiza sus esquemas para, en un último paso, adquirir aquella información que considera relevante de los medios (Kosicki y Mcleod, 1990). De esta manera se desestima la información irrelevante y se construye una visión de la realidad en función de la experiencia personal, la interacción con sus iguales y la interpretación selectiva de la información extraída de los medios (Neuman et al., 1992).

Pero también los medios de comunicación pueden ser influenciados por fuentes externas. McCombs explica que los medios de comunicación reciben una sustancial influencia de otros medios de comunicación de importancia similar o superior (McCombs y Shaw, 1972). En esta influencia hay que considerar otros actores políticos, institucionales, etc., junto a la de los medios de comunicación.

Este fenómeno se ha visto incrementado desde la aparición de Internet (Curtin y Gaither, 2003). Según ciertos estudios, las jóvenes generaciones de usuarios "nativos" de Internet son menos influenciables por los medios de comunicación de masas y son más proclives a conseguir la información desde la red (Coleman y McCombs, 2007). Internet permite a sus usuarios ser menos influenciables por el establecimiento de determinados mensajes en los medios tradicionales y les permite asimismo influir en otros usuarios facilitándoles el acceso a fuentes y contenidos de interés. También hay estudios que demuestran que la Agenda de Internet influye en la Agenda de los medios de comunicación (Jeong, Kim y Shin, 2008; Aikat, 2008). Los estudios recientes sostienen que la relación establecida hace décadas está siendo sometida a presiones por los nuevos medios (Gurevitch, Coleman, and Blumler, 2009). Coleman y McCombs (2007) han encontrado que la diferencia de uso de los medios entre diferentes generaciones ha interrumpido el poder de los medios en el establecimiento de la agenda.

\section{EL POSICIONAMIENTO DE LA PRENSA ANTE EL HECHO NOTICIOSO}

Los medios de comunicación y los periodistas estarán en cierta medida sujetos al posicionamiento de los grupos inversores que controlan los medios, con poco margen para definir cuáles son los temas de interés de ese momento (Gavaldà, 1996; Reig, 2011). Dado que los medios de comunicación encuadran la realidad dependiendo del discurso narrativo de sus grupos de interés, tratarán de manera distinta situaciones de conflicto social. Así, ante una realidad de conflicto social, ante un hecho concreto, se establecerán diversas arquitecturas narrativas en las que se dará una determinada interpretación.

Desde una perspectiva instrumental se entiende que hay una intencionalidad por parte del emisor a la hora de jerarquizar los contenidos de una manera u otra. Esta intención viene determinada por la búsqueda de objetivos por parte de los periodistas o los medios de comunicación, hipótesis esta que asumimos como propia en esta investigación (H. M. Kepplinger, 1989). Esta teoría, por su parte, se ve enriquecida por una aportación posterior que introduce el concepto de Key Event, suceso que centra la atención de periodista y público, y que modifica el criterio de selección de 
las noticias, incrementando el número y la frecuencia de noticias relacionadas (Kepplinger y Habermeier, 1995).

\section{LAS REDES SOCIALES Y TWITTER COMO MEDIOS DE INFORMACIÓN}

Internet es un factor clave en la sociedad actual del conocimiento, puesto que facilita el intercambio y la creación de contenidos culturales e informativos. Esta característica se ha visto potenciada por el auge de las redes sociales y la penetración de uso de los Smartphones. En palabras de Juan José Prieto:

Una red social se define como una organización o estructura generada a través de las relaciones de diferentes actores (personas, instituciones, organizaciones, sociedades, etc.), debiendo poseer o estar vinculadas a ciertas particularidades o rasgos comunes con el fin de poder interactuar entre sí. (Prieto Gutiérrez, 2011).

El paradigma de estas redes sociales es la interacción entre los usuarios y la creación colectiva de los contenidos. Dado que la conversación es el modo de creación de dichos contenidos, se utilizan en ella mecanismos como la convergencia de usuarios en determinados "hilos de conversación". También se utiliza la inclusión de escritos de información u opinión. En definitiva, la sindicación de contenidos por aspectos de interés colectivo. Por dichas características, la medición y observación de estas redes sociales, tanto cualitativa como cuantitativamente, son primordiales para definir realmente el grado de influencia de los contenidos, los actores que participan en el proceso, la forma de emitir los mensajes y el alcance real de los mismos.

En un estudio realizado por ComScore se constata que el $82 \%$ de la población online accede de manera intensiva a las redes sociales, pasando en ellas un $20 \%$ del tiempo total online de los internautas a nivel mundial en 2011, 14 puntos más que las cifras de 2007 (ComScore, 2012). Además, y según un estudio de IAB Spain, el $59 \%$ de los usuarios de redes sociales consulta bastante o muy frecuentemente noticias en portales y/o periódicos online.

Las relaciones en redes sociales se combinan para crear una comunidad informal, una red social con valores y normas compartidas (Jones, 2008). La forma en que la comunidad se relaciona con y responde a los principales medios de comunicación sigue siendo poco explorada, especialmente en el contexto de las controversias políticas.

La idea de que una red social puede actuar como una nueva esfera pública también se está considerando cada vez con más fuerza. La interacción y discusión facilitada por el uso, su inmediatez, su carácter público y la posibilidad de adjuntar contenidos multimedia a los contenidos textuales, ofrece un foro de gran potencial para la esfera pública digital (Milliken y O’Donnell 2008). Este foro, además, da la posibilidad a opiniones minoritarias de darse a conocer a más personas, cosa que con los medios tradicionales era imposible (Silverstone, 1999, 2001; Downey y Fenton, 2003). Este potencial intercambio de opiniones diferentes puede estar derivando a una concatenación de monólogos según los gustos, apetencias y opiniones políticos en las redes sociales, por lo que es interesante explorar estas dinámicas para comprender las posibilidades de este foro de discusión e información y si realmente está actuando como catalizador de la discusión sobre la esfera pública (Sayre, Bode, Shah, Wilcox y Shah, 2010). 
En el ámbito político, para la mayoría de votantes, los medios de comunicación tradicionales siguen siendo primordiales y, por tanto, continúan siendo la mayor fuerte de información. Pero también es posible que la presencia y la influencia de los nuevos medios de comunicación social amplifiquen la importancia de determinados contenidos en la jerarquización de noticias.

Twitter es un sistema gratuito de microblogging. En él se publican mensajes (tweets o tuits, en su adaptación al castellano) de 140 caracteres como máximo. Se compone de seguidores (followers) y seguidos (following), siempre desde una perspectiva subjetiva. Estos dos colectivos no necesariamente coinciden. El usuario se define por llevar delante del nick una @. Si en un mensaje que emitimos se utiliza la arroba (ejemplo: @usuario) ese mensaje se está refiriendo directamente a dicho usuario. El retweet, retuit o RT permite difundir el mensaje emitido por otro usuario a tu comunidad de seguidores. El hashtag se configura añadiendo \# al término (contenido en el mensaje) con el que queremos etiquetar el tweet, lo que facilita su posterior búsqueda. La repetición masiva de dichos hashtags o asuntos, hace que se configuren los temas candentes o trending topics. Twitter, asimismo, permite la mensajería directa privada a usuarios que se siguen entre ellos.

Los usos de Twitter cambian con el tiempo. Permite, por ejemplo, la retransmisión de un acontecimiento en tiempo real. También permite establecer una canalización del activismo político o ciudadano, por lo que se ha creado el término "ciberactivista". Permite, por último, compartir conocimiento, información, emisiones corporativas o difusión de noticias. En un primer momento, Twitter se utilizó como un método de compartir estados de ánimo y actividades (Java et al., 2007). Pronto se convirtió en una herramienta para buscar y compartir información con un sistema de recomendación, para la que se han habilitado diferentes herramientas subsidiarias (Phelan, Owen; McCarthy, Kevin; Smyth, Barry, 2009). Se establecen así tres tipos de usuarios primarios: las fuentes de información, que suelen tener un gran número de seguidores; los "amigos", que suelen ser personas relacionadas con el usuario por un cierto grado de cercanía (familiar, relacional, geográfica, ideológica o de intereses profesionales) y los buscadores de información, aquellos que rara vez emiten pero que siguen a gran cantidad de personas (Java et al., 2007). También se están realizando estudios acerca de la personalidad de aquellos que prefieren utilizar Twitter a Facebook, demostrando que según la personalidad, se le da diferente uso a cada una de estas redes (Hughes, Rowe, Batey y Lee, 2012).

Desde esta perspectiva, podría pensarse que el funcionamiento de Twitter es completamente viral y que abre enormes posibilidades a medios de comunicación, empresas y partidos políticos para difundir de una manera efectiva. Sin embargo, estudios realizados sobre las interacciones entre usuarios reflejan que dicho funcionamiento es básicamente lineal, existiendo determinados individuos que actúan en forma de nodos (Huberman, Romero y Wu, 2008). De hecho, la barrera para que un tweet sea popular está en el primer retweet. Una vez realizado este primer salto, el tweet es retwiteado en un $2 .^{\circ}, 3 .^{\circ}$ y $4 .^{\circ}$ nivel de distancia casi inmediatamente, lo que revela un alto grado de homofilia entre los usuarios de Twitter (Kwak, Lee, Park y Moon, 2010). Estos contenidos serán más creíbles para el usuario porque los ha emitido alguien a quien ha decidido seguir (Zhao y Rosson, 2009). Estas características 
además, conceden a la herramienta un alto potencial como medio conversacional y colaborativo (Honeycutt y Herring, 2009).

A la hora de medir la influencia en Twitter, es conveniente distinguir tres indicadores principales: número de seguidores, número de retweets y número de menciones. El número de seguidores no indica necesariamente un número alto de retweets y menciones. Un alto número de seguidores indica la popularidad del titular de la cuenta y tiene su significación en el número de menciones. Sin embargo, el número de retweets viene condicionado por el interés del tweet (Cha, Haddadi, Benvenuto y Gummadi, 2010).

Twitter, que está basado en la relación y la interacción de los usuarios, tiene la capacidad de crear comunidades de aprendizaje colaborativo, fundamentadas en la puesta en común de contenidos y en la recomendación de información (Lewis, Pea y Rosen, 2010). Esta colaboración se ha visto reflejada en las convocatorias realizadas para movilizaciones sociales que no dependían nominalmente de ningún organismo coordinado. En un reciente trabajo aparecido en Scientific Reports se comprueba mediante el posicionamiento geográfico de los usuarios tempranos o convocantes de las movilizaciones del 15M en España, y de los transmisores y difusores de las mismas, que hay una correlación importante entre las redes sociales, el contagio social y las dinámicas colectivas, lo que favorece el reclutamiento de participantes en protestas (González-Bailón, Borge-Holthoefer, Rivero y Moreno, 2011).

Las redes sociales crean una sensación de poder estar conectado con cualquier parte del mundo. Pero los análisis que miden el número de interacciones respecto a variables como distancia geográfica, lengua o país, reflejan que dicha interacción se da mayoritariamente entre usuarios que comparten lugar de situación u otras características específicas comunes (Takhteyev, Gruzd y Wellman, 2012).

Desde 2004 la situación ha cambiado radicalmente con la aparición en el modelo informativo de las redes sociales y su permanente auge de usuarios, en continuo ascenso desde 2005. Para Campos Freire (2008):

Esas relaciones y redes sociales o profesionales establecidas y desarrolladas a través de Internet nos sitúan ante una nueva fase, que algunos califican como postmediática, de una sociedad de servicios aún mucho más acelerada y en la que la atención aparece más segmentada, personalizada, instantánea, diluida, convergente, transparente, flexible, liviana, conversacional, interconectada y abocada a la colaboración, participación y trivialización. Las relaciones de los públicos con los medios están cambiando: crece la fragmentación y se diluye la mediación.

Pese a que en los resultados de su investigación, Campos Freire reconoce que las redes sociales obtienen los contenidos informativos de los medios de comunicación tradicionales, también introduce elementos novedosos de análisis como el periodismo 3.0, periodismo colaborativo y periodismo social o ciudadano. La influencia de la cultura colaborativa, de la cultura "wiki"1, ha llegado a casi todos los ámbitos de Internet, en particular en las redes sociales.

1 Wiki: sitio web cuyas páginas pueden ser editadas por múltiples voluntarios a través del navegador web. http://es.wikipedia.org/wiki/Wiki 
El periodismo ha asumido un potente rol en la estructuración social, política y de opinión pública. Actúa de una manera activa y no meramente de canal transmisor de información. Tradicionalmente se había dado un sistema de reciprocidad entre los periodistas y los políticos, basado en un sistema de negociación constante que permitía, en una sociedad mediatizada, difundir, encuadrar, jerarquizar y modelar la acción política de una determinada sociedad (Casero Ripollés, 2008). Las redes sociales, y en especial Twitter, han variado esta situación. Políticos y periodistas conciben Twitter como una plataforma de líderes de opinión y sus "temas calientes" como aspectos a tener en cuenta en el tratamiento mediático y político ${ }^{2}$. Mediante la observación de los Trending Topics de Twitter se ha llegado a la paralización de iniciativas legislativas como la aprobación de la denominada "Ley Sinde" en diciembre de $2011^{3}$. Pero la relación entre los periodistas, medios de comunicación y Twitter es compleja.

A pesar de que los medios han empezado a tener en cuenta la participación de los usuarios, en la práctica no se facilitan mecanismos para que la audiencia genere contenidos informativos en los medios de comunicación. Esta prerrogativa sigue estando casi exclusivamente en manos de los periodistas, dejando a los ciudadanos una labor de opinión y comentario sobre los contenidos generados (SánchezGonzález y Alonso, 2012). Pero los periodistas se ven influidos por la interacción con el ciudadano, dado que:

la sola existencia de la acción informativa directa de los ciudadanos genera modificaciones en la acción profesional de los periodistas: otras modalidades de reporteo, nuevas formas de verificación y, sobre todo, un proceso de selección mucho más preciso para evitar que la marea informativa ahogue en definitiva la capacidad de comprensión de los sucesos y disgregue de modo irreversible a las audiencias (Pellegrini, 2010, p. 276).

Sin embargo, Twitter se ha revelado como una herramienta crucial en la cobertura inmediata de acontecimientos de alta intensidad y diseminación informativa como desastres naturales o sucesos traumáticos socialmente (Li y Rao, 2010). Según el eurobarómetro Journalists and Social Media, de $2012^{4}$, la mayor parte de los periodistas entrevistados, utiliza los medios sociales como herramienta en su trabajo, sobre todo Facebook y Twitter. Los periodistas las utilizan como un recurso para conseguir información. En el caso de Twitter, se utiliza principalmente para conseguir información internacional. Las redes sociales les sirven para estar al día de lo que sucede, para crear conexiones con personajes clave y con las audiencias, para promocionar contenidos propios del medio en el que trabajan y para buscar material gráfico. La mayoría de los periodistas utiliza también las redes sociales en su vida personal. Lo hacen para ser más creíbles y naturales ante su audiencia.

La mayoría tiene claro que su uso de las redes sociales se incrementará en los próximos meses, algunos por la presencia de eventos clave en su país como elecciones, pero la mayoría porque piensan que las redes sociales tendrán cada vez más impor-

2 http://sociedad.elpais.com/sociedad/2011/12/29/actualidad/1325164747_060100.html.

$3 \mathrm{http}: / /$ politica.elpais.com/politica/2011/12/05/actualidad/1323100120_8̄11714.html.

$4 \mathrm{http} / / /$ ec.europa.eu/public_opinion/archives/quali/journsm_en.pdf. 
tancia en su trabajo. Consideran beneficiosa la aparición de estas herramientas, entre otros aspectos, por su rapidez e instantaneidad, porque facilitan el acceso a la información a los usuarios, permiten el acceso a variedad de opiniones, porque refuerzan y fidelizan audiencias y otorgan credibilidad y autenticidad.

Sin embargo, en cuanto se les pregunta por la capacidad de influencia de las redes sociales, la división es total entre los que creen que las redes influyen determinantemente en la opinión pública y los que piensan que sigue siendo la televisión la que realmente la configura.

\section{HIPÓTESIS DE TRABAJO}

A la vista de todo lo expuesto anteriormente, se plantean las siguientes hipótesis de trabajo:

1. Se ha realizado una difusión exhaustiva por parte de los medios de comunicación de las movilizaciones en contra de los recortes en educación acaecidas en Valencia desde el día 15 de febrero hasta el día 1 de marzo de 2012.

2. Los medios de comunicación han recogido la mayoría de las consignas de los convocantes de las manifestaciones en las cuentas oficiales de los periódicos.

3. Los medios de comunicación han incluido en su timeline las publicaciones de las cuentas personales de periodistas en Twitter. Twitter.

4. Ha existido un porcentaje significativo de artículos con menciones a

5. Se han incluido contenidos aparecidos en Twitter no propios en la cobertura de las noticias relacionadas en las cuentas de los medios de comunicación.

Para realizar el presente estudio se realizó un análisis de contenido de los artículos relacionados con los acontecimientos acaecidos en la ciudad de Valencia a raíz de las protestas estudiantiles. Se analizaron los artículos aparecidos en las ediciones digitales de la prensa regional valenciana de mayor tirada durante las fechas comprendidas entre el 15 de febrero y el 1 de marzo de 2012. Se cuantificó su difusión directa en Twitter. Asimismo, se realizó un análisis cuantitativo de los tweets o mensajes emitidos por las cuentas oficiales de dichos medios de comunicación en la red de microblogging Twitter, relacionándolos con los contenidos aparecidos en las ediciones digitales de los diarios. Por último, se realizó un análisis de contenido de las cuentas de Twitter de los medios de comunicación y de los mensajes que aparecen en el timeline de las mismas. Se analizaron las interacciones y la difusión de los mensajes. Se estudió la relación entre la presencia del contenido multimedia y la redifusión del mensaje.

También se cuantificó la incidencia global de las etiquetas o hashtags que se utilizaron mayoritariamente para difundir y realizar el seguimiento de las movilizaciones y reacciones sociales y políticas a los acontecimientos. Se realizó una valoración de la incidencia de dichas etiquetas en los artículos aparecidos en los medios de comunicación anteriormente mencionados. 


\section{CONCLUSIONES Y DISCUSIÓN}

Twitter ha cambiado el modelo de generación de noticias en la cobertura del suceso en los periódicos seleccionados. Además, aumenta el volumen de noticias incluyendo al servicio de los medios de comunicación en su edición digital el análisis de la repercusión de los sucesos en las redes sociales.

El seguimiento se hace exhaustivo en las ediciones digitales de los diarios a análisis en el momento en el que aparece un Key Event. Éste se puede localizar en la tarde del día 20 de febrero, pero viene precedido de las respuestas policiales a las protestas de los días 15 y 16 de febrero. Su repercusión se puede observar por la subida de uso de los hashtags principales, el volumen de artículos publicados en las ediciones digitales y en la frecuencia de tweets emitidos por parte de los medios. Este Key Event posiciona y fija la noticia en la Agenda Setting de los medios analizados, haciendo que su cobertura y presencia en la edición digital se mantenga constante, aunque descendente, desde el día 20 de febrero hasta el día 1 de marzo.

Noticias publicadas entre el 15 de febrero y el 1 de marzo de 2012

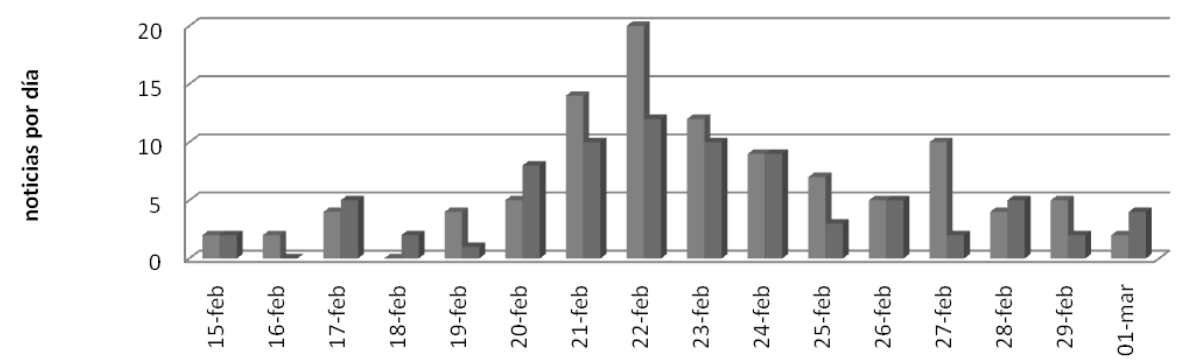

\begin{tabular}{|c|c|c|c|c|c|c|c|c|c|c|c|c|c|c|c|c|}
\hline & $15-\mathrm{fe}$ & $16-f e$ & $17-\mathrm{fe}$ & $18-f e b$ & $19-f e b$ & $20-\mathrm{feb}$ & $21-\mathrm{feb}$ & $22-\mathrm{feb}$ & $23-\mathrm{feb}$ & $24-f e b$ & 25 -feb & $26-f e b$ & $27-f e b$ & $28-\mathrm{feb}$ & $29-\mathrm{feb}$ & $\begin{array}{l}\text { 01- } \\
\text { mar }\end{array}$ \\
\hline Levante & 2 & 2 & 4 & 0 & 4 & 5 & 14 & 20 & 12 & 9 & 7 & 5 & 10 & 4 & 5 & 2 \\
\hline - Las Provincias & 2 & 0 & 5 & 2 & 1 & 8 & 10 & 12 & 10 & 9 & 3 & 5 & 2 & 5 & 2 & 4 \\
\hline
\end{tabular}

Gráfico 1: Noticias publicadas en los medios analizados durante el periodo de análisis.

Fuente: Elaboración propia 


\section{Tweets publicados del 15 de febrero al 1 de marzo de 2012}

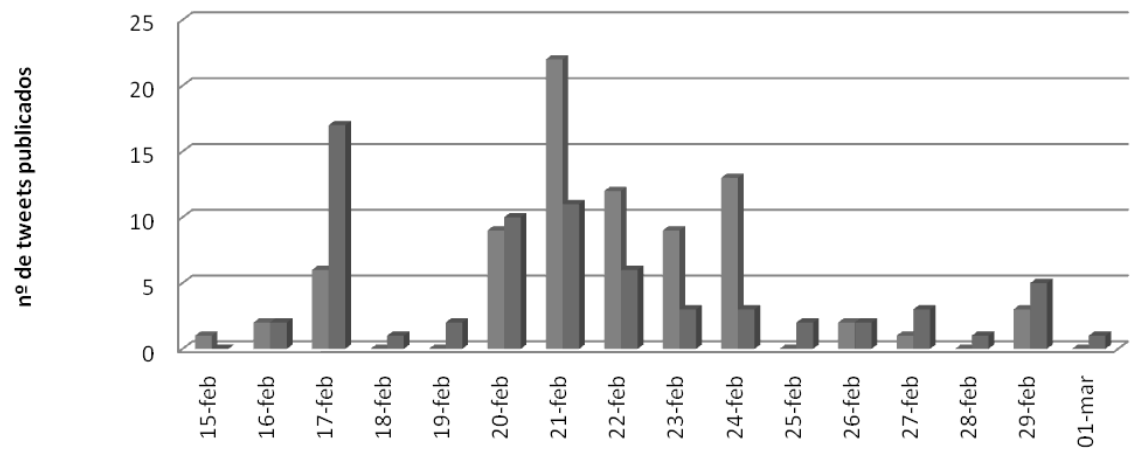

\begin{tabular}{|c|c|c|c|c|c|c|c|c|c|c|c|c|c|c|c|c|}
\hline & $\begin{array}{l}15- \\
\text { feb }\end{array}$ & $\begin{array}{l}16- \\
\text { feb }\end{array}$ & $\begin{array}{l}17- \\
\text { feb }\end{array}$ & $\begin{array}{l}18- \\
\text { feb }\end{array}$ & $\begin{array}{l}19- \\
\text { feb }\end{array}$ & $\begin{array}{l}20- \\
\text { feb }\end{array}$ & $\begin{array}{l}21- \\
\text { feb }\end{array}$ & $\begin{array}{l}22- \\
\text { feb }\end{array}$ & $\begin{array}{l}23- \\
\text { feb }\end{array}$ & $\begin{array}{l}24- \\
\text { feb }\end{array}$ & $\begin{array}{l}25- \\
\text { feb }\end{array}$ & $\begin{array}{l}26- \\
\text { feb }\end{array}$ & $\begin{array}{l}27- \\
\text { feb }\end{array}$ & $\begin{array}{l}28- \\
\text { feb }\end{array}$ & $\begin{array}{l}29- \\
\text { feb }\end{array}$ & $\begin{array}{l}\text { 01- } \\
\text { mar }\end{array}$ \\
\hline Levante & 1 & 2 & 6 & 0 & 0 & 9 & 22 & 12 & 9 & 13 & 0 & 2 & 1 & 0 & 3 & 0 \\
\hline Las Provincias & 0 & 2 & 17 & 1 & 2 & 10 & 11 & 6 & 3 & 3 & 2 & 2 & 3 & 1 & 5 & 1 \\
\hline
\end{tabular}

Gráfico 2: Tweets emitidos en las cuentas analizadas durante el periodo de análisis. Fuente: Elaboración propia

\begin{tabular}{|c|c|c|c|c|c|c|c|c|c|}
\hline & \multicolumn{7}{|c|}{ sección } & \multirow[t]{2}{*}{ Total } \\
\hline & & local & opinión & $\begin{array}{l}\text { Cartas de } \\
\text { lectores }\end{array}$ & economía & política & \begin{tabular}{|l} 
televisió \\
$\mathrm{n}$
\end{tabular} & sociedad & \\
\hline \multirow[b]{2}{*}{ medio } & Levante & 59 & 31 & 13 & 1 & 0 & 1 & 0 & 105 \\
\hline & $\begin{array}{l}\text { Las } \\
\text { provincias }\end{array}$ & 57 & 6 & 12 & 0 & 4 & 0 & 1 & 80 \\
\hline Total & & 116 & 37 & 25 & 1 & 4 & 1 & 1 & 185 \\
\hline
\end{tabular}

Tabla 1: Artículos publicados por sección. Fuente: Elaboración propia

Asimismo, a la luz de los datos obtenidos se puede comprobar el ciclo corto de la atención de sucesos noticiosos en Twitter. Existe un rápido descenso de hashtags de uso multitudinario como los analizados \#primaveravalenciana e \#IESLluisVives. Esto refleja la rápida pérdida de interés de los públicos ante la cantidad de información que circula a través de Twitter. 


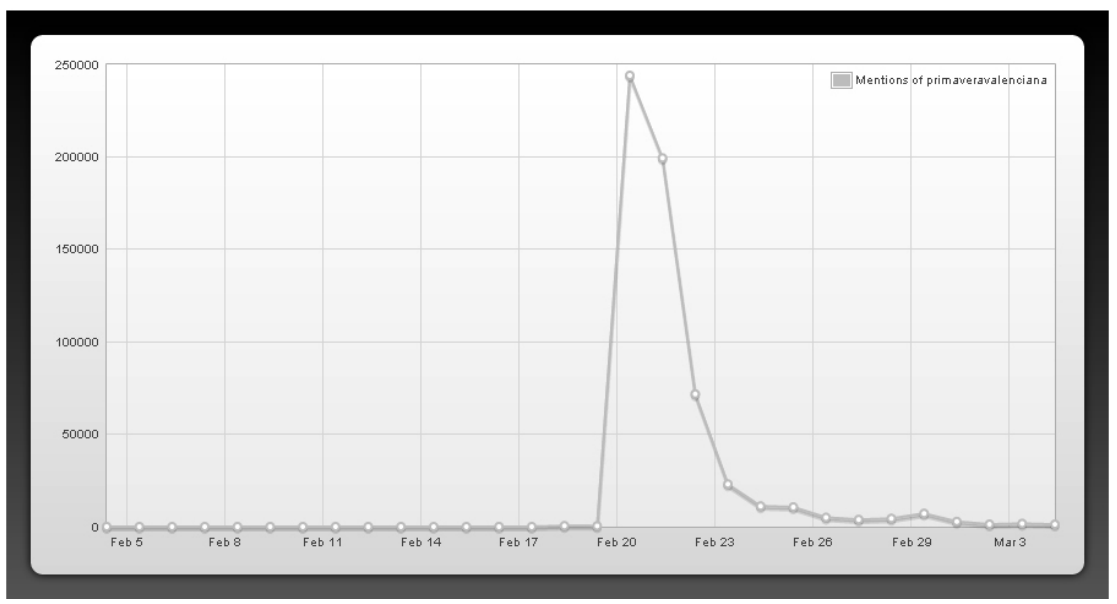

Gráfico 3: Utilización global del hashtag \#primaveravalenciana. Fuente: Topsy

Corrobora la teoría del Framing el importante peso relativo que tiene la difusión de las reacciones ante la noticia y, sobre todo en el caso de Levante EMV, la importancia de los artículos de opinión que encuadran y analizan los elementos noticiosos acaecidos. Pero también se comprueba que los medios de comunicación apenas hacen uso de la red de microblogging Twitter para dar a conocer la línea editorial o las opiniones de sus articulistas o lectores.

Los medios utilizan la herramienta Twitter de una manera desigual y con unos usos muy determinados. Mediante la referencia a otras cuentas envían corresponsales a las movilizaciones y consiguen material audiovisual que posteriormente utilizan en galerías de fotos y en videos tomados por periodistas y participantes en las movilizaciones e incrustados en sus ediciones digitales. Estas noticias con contenidos audiovisuales tienen, a la luz de los datos obtenidos, una mayor posibilidad de redifusión a través de las redes sociales y de Twitter. Asimismo se hacen eco de las repercusiones que las movilizaciones provocan en las redes sociales, utilizando la herramienta como fuente de consulta para la redacción de artículos posteriores al suceso noticioso. Así convierten las repercusiones a su vez en noticia y mantienen el foco informativo sobre el suceso.

La difusión a través de las cuentas oficiales de Twitter es inferior a la difusión que se hace desde los widgets insertados en las páginas de las noticias. Esta situación demuestra poca proactividad por parte de los gestores de las cuentas oficiales de los medios de comunicación, que dejan en manos de los usuarios la potestad de compartir más o menos una noticia. 


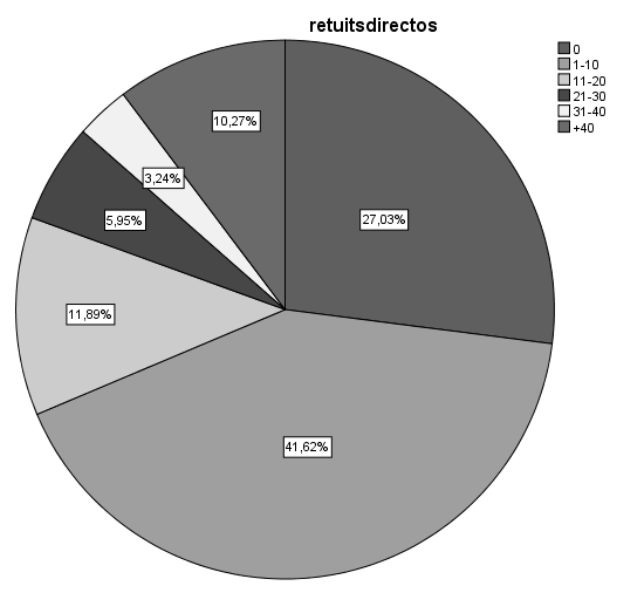

Gráfico 4: Difusión desde widgets sociales. Fuente: elaboración propia

Salvo en casos muy concretos, desde las cuentas oficiales, apenas utilizan la interacción con otros usuarios. Tampoco remiten a personal ajeno a la nómina del medio de comunicación. De esta manera se pierde gran parte de las potencialidades de la herramienta Twitter de generación de información y conocimiento colaborativo.

Una de las potencialidades que no se utilizan de manera óptima es el etiquetado de las publicaciones en Twitter. Solo el $41^{\prime} 61 \%$ de los mensajes emitidos se etiqueta con los hashtags específicos del suceso noticioso. Esto hace que el 58 '39\% restante quede fuera del buscador de Twitter.

La relación entre el número de menciones a los hashtags estudiados y la cantidad de tweets emitidos por las cuentas de los medios de comunicación resulta evidente. Durante las movilizaciones del día 17, uno de los días de mayor incidencia del hashtag \#IESLluis Vives, la cuenta oficial del diario @lasprovincias aumenta significativamente el número de tweets emitidos. Por su parte, @levante_emv actúa de la misma manera en el periodo de mayor incidencia de \#primaveravalenciana. Aunque @ lasprovincias no actúa de la misma manera que @levante_emv en este segundo caso, desde las 13:37 horas del día 17 de febrero comienza a remitir a las cuentas personales de los periodistas destacados.

Como líneas futuras de investigación se plantean varios interrogantes a la luz de los datos. En primer lugar si se ha establecido una narrativa colectiva en Twitter en la transmisión de las informaciones y el grado de influencia en los contenidos periodísticos fruto de la interacción con usuarios en esta red de información. Esta narrativa se realizará mediante un estudio comparativo entre el contenido y el discurso de los mensajes de las cuentas de los periodistas y las informaciones aparecidas en las ediciones digitales de los diarios.

Asimismo, mediante un análisis de discurso de los artículos seleccionados, sería conveniente realizar una comparativa sobre el tono de los mensajes en Twitter de los periodistas, creando una taxonomía de los mismos y relacionándolos con la Teoría 
del Encuadre Mediático o Framing, para corroborar si el posicionamiento editorial es compartido por los periodistas en las redes sociales o si difiere sustancialmente.

Por último, viendo que la presencia de las redes sociales y de Twitter en la cobertura y confección de las noticias y en la difusión de las mismas es un hecho, sería interesante realizar un estudio sobre otro elemento noticioso similar posterior para establecer los índices de crecimiento o decrecimiento del uso de esta herramienta.

\section{BIBLIOGRAFÍA}

AIKAT, D. (2008). "The Blending of Traditional Media and the Internet: Patterns of Media Agenda Setting and Web Search Trends Before and After the September 11 Attack", Annual Meeting of the International Communication Association (New York, NY: October 10, 2008).

Aouragh, M., Alexander, A., (2011). The Egyptian experience: sense and nonsense of the Internet revolution. International Journal of Communication 5, 1344-1358.

CARTY, V., (2010). New information communication technologies and grassroots organization. Information, Communication and Society 13 (2), 155-173.

CASERo Ripollés, A. (2008). Modelos de relación entre periodistas y políticos: La perspectiva de la negociación constante. Estudios sobre el Mensaje Periodístico, 14 pp. 111-128.

Castells, M. (2009). Comunicación y poder. Madrid. Alianza Editorial.

Cha, Haddadi, Benvenuto y Gummadi, (2010). Measuring User Influence in Twitter: The Million Follower Fallacy. Proceedings of the Fourth International AAAI Conference on Weblogs and Social Media.

Coleman, R. y McCombs, M. (2007). "The Young and Agenda-Less? Exploring Age-related Differences in Agenda Setting on the Youngest Generation, Baby Boomers, and the Civic Generation", Journalism \& Mass Communication Quarterly 84 (Autumn 2007): 495-508.

Curtin, P. y Gaither, K. (2003). "International Agenda-Building in Cyberspace: A Quantitative Content Analysis of Middle East Government English-Language Web Sites", Annual Meeting of the International Communication Association: 1-58.

DE GRACIA, A. (2011). Las rebeliones árabes sientan bases históricas por el uso de la tecnología. CIC Cuadernos de Información y Comunicación, vol. 16 pp. 167-174.

Downey, J., y Fenton, N. (2003). "New Media, Counter Publicity and the Public Sphere." New Media \& Society 5 (2): 185-202.

Earl, J., Kimport, K., Prieto, G., Rush, C., Reynoso, K. (2010). Changing the World one webpage at a time: conceptualizing and explaining Internet Activism. Mobilization: An International Journal 15 (4), 425-446.

Fisher, D., BoekKoOI, M. (2010). Mobilizing friends and strangers: understanding the role of the Internet in the step it up day of action. Information, Communications and Society 13 (2), 193-208.

FueNTES RodRíGuez, C. (2010) Ideología e imagen: la ocultación en la prensa de la violencia social o lo políticamente correcto. Discurso \& Sociedad. Vol. 4(4) 2010, 853-892.

FUMERO-REVERÓN, A. (2011). IRC 2.0. Medios para la información, la relación y la comunicación en la web 2.0. El profesional de la información, noviembre-diciembre, v. 20, n. 6, pp. 605-609. http://dx.doi.org/10.3145/epi.2011.nov.01. 
Galtung, J. y Ruge, M. (1965). "The structure of foreign news: The presentation of the Congo, Cuba and Cyprus crises in four foreign newspapers”. Journal of International Peace Research, vol. 1, pp. 64-90

Gurevitch, M., Coleman, S. y Blumler, J. G.. (2009). "Political Communication: Old and New Media Relationships". Annals of the American Academy of Political and Social Science 625 (1): 164-181.

Honeycutt, C. y Herring, S. C. (2009). Beyond microblogging: Conversation and collaboration via Twitter. Proceedings of the Forty-Second Hawai'i International Conference on System Sciences (HICSS-42). Los Alamitos, CA: IEEE Press.

Huberman, Bernardo A., Romero, Daniel M. y Wu, Fang, (2008) Social Networks that Matter: Twitter Under the Microscope.

Hughes, D.J., Rowe, M., Batey, M. y Lee, D. (2012). A tale of two sites: Twitter vs. Facebook and the personality predictors of social media usage. Computers in Human Behavior 28, 561-569.

Humanes, M. L. (2001). El encuadre mediático de la realidad social. Un análisis de los contenidos informativos en televisión. Euskal Herriko Unibertsitatea.

Java, A., Finin, T., Song, X. y Tseng, B. (2007). Why We Twitter: Understanding Microblogging Usage and Communities. Proceedings of the 9th WebKDD and 1st SNAKDD 2007 workshop on Web mining and social network analysis.

JeOng, Y., KIM, K., y ShIN, W. (2008). "Agenda Building Function of Internet Searches: Measuring the Unique Contribution of the Public Agenda on the Media Agenda", Annual Meeting of the International Communication Association (New York, NY: October 10, 2008);

Jones, Sydney. (2008). “Anthropology of YouTube". Pew Internet and American Life Project

Kepplinger, H. M. y Habermeier, J. (1995). "The impact of Key Events on the presentation of reality", European Journal of Communication, 10, pp. 371-390.

Kotler, P. (1986). "The prosumer movement: a new challenge for marketers". In Advances in Consumer Research, Volume 13, eds. Richard J. Lutz, Provo, UT. Association for Consumer Research, Pages: 510-513.

Kwak, H., Lee, C., Park, H. y Moon, S. (2010). What is Twitter, a social network or a news media? Proceedings of the 19th international conference on World wide web.

Lewis, Pea y Rosen (2010). Co-creation of meaning using mobile social media. Social Science Information, 0539-0184; Vol. 49(3): 1-19; 370726.

LI, Y. y RAO, H.R. (2010). Twitter as a rapod response news service: an exploration in the context of the 2008 China earthquake. EJISDC 42, 4, pp. 1-22

McCombs, M. y Shaw, D. (1972). The Agenda Setting Function of Mass Media, Public Opinion Quarterly 36: 176-187.

- (1993). The evolution of agenda-setting research: Twenty-five years in the market place of ideas, en Journal of Communication, vol. 43, núm. 2

McCombs, M., Shaw, D. y WeAver, D. (1997). Communication and democracy. Exploring the intellectual frontiers in agenda-setting theory. Mahwah, NJ: Erlbaum.

Milliken, M., y O’Donnell, S. (2008). "User-Generated Online Video: The Next Public Sphere?" IEEE International Symposium on Technology and Society (ISTAS 08), Fredericton, NB, May 24-26.

Morozov, E., (2010), february 20. The digital dictatorship. The Wall Street Journal.

Neuman, R. W., Just, M. R. y CRigler, A. N. (1992). Common knowledge. News and the construction of political meaning. Chicago: University of Chicago Press. 
Pellegrini, S. (2010). Análisis conceptual del periodismo ciudadano y propuesta metodológica para analizar su contribución informativa. Palabra Clave, 13 (2), 271-290.

Phelan, O.; McCarthy, K.; Smyth, B. (2009). Using Twitter to recommend real-time topical news. Proceedings of the third ACM conference on Recommender systems.

Prieto Gutiérrez, J. J. (2011). Herramientas para el análisis y monitoreo en Redes Sociales. International Review of Information Ethics n. ${ }^{\circ} 12,33-40$.

Rubio Ferreres, J. M. (2009). Opinión pública y medios de comunicación. Teoría de la 'Agenda Setting'. En: Gazeta de Antropología, No 25 /1, 2009, Artículo 01.

SÁncheZ-GonzÁlez, M. y Alonso, J. (2012): "Propuesta metodológica para el análisis de las tecnologías de participación en cibermedios", en Revista Latina de Comunicación Social, 67. La Laguna (Tenerife): Universidad de La Laguna, páginas 148 a 178 recuperado el 9 de Abril de 2012, de http://www.revistalatinacs.org/067/ 951_Malaga/07_Sanchez.html DOI: 10.4185/RLCS-067-951-148-178 / CrossRef link.

SArtori, G. (1992). "Opinión Pública", en G. Sartori (Ed.), Elementos de Teoría Política. Madrid: Alianza Singular.

SCHEUfELE, D. (1999). Framing as a theory of media effects. Journal of Communication. Volume 49, Issue 1, pages 103-122, March 1999.

Scheufele, DA, Tewksbury D. (2007). Framing, Agenda Setting, and priming: The evolution of three media effects models. Journal of Communication. 57:9-20, Number 1.

ScHulz, W. (1982): "News structure and people awareness of political events", Gazzette, 30, pp. $139-153$.

Silverstone, R. (1999). Why Study the Media? Thousand Oaks, CA: Sage Publications.

- (2001). "Finding a Voice: Minorities, Media and the Global Commons". Emergences: Journal for the Study of Media \& Composite Cultures 11 (1): 13-27.

TAKhteYev, Y., Gruzd, A., y Wellman, B., (2012). Geography of Twitter networks. Social Networks 34 pp. 73- 81.

TAN, Y. Y WeAVER, D. H. (2007). Agenda Setting effects among the media, the public, and Congress, 1946-2004. Journalism and Mass Communication Quarterly, 84(4), 729744.

Tuchman, G. (1978). Making news. A study in construction of reality. New York, Free Press. Wolf, Mauro, Los efectos sociales de los media, Editorial Paidós, Barcelona, 1994.

\title{
RESUMEN
}

Twitter cambia la relación entre los medios de comunicación de masas y sus públicos. Por tanto cambia también el proceso de formación de la Opinión Pública. En este artículo se analiza la presencia de Twitter en las noticias relacionadas con la "Primavera valenciana" y cómo ha influido de manera relevante en el establecimiento de la Agenda Setting.

Palabras clave: periodismo, redes sociales, Twitter, Agenda Setting, Key Event.

\section{Twitter, \#primaveravalenciana and generation of news}

\begin{abstract}
Twitter changes the relationship between the mass media and their audiences. Therefore also changes the process of forming Public Opinion. This article analyzes the presence of Twitter in the news related to the "Primavera valenciana" and how it has significantly affected the establishment of Agenda Setting.
\end{abstract}

Keywords: journalism, social network, Twitter, Agenda Setting, Key Event. 